\title{
MAXIMAL SUBSEMIGROUPS OF LIE GROUPS THAT ARE TOTAL
}

\author{
by JIMMIE D. LAWSON
}

(Received 19th September 1986)

The major problem with which this paper is concerned is determining criteria that allow one to decide whether the subsemigroup generated by a subset $B$ of a group $G$ is all of $G$. Motivations for considering this problem arise from at least two sources.

The first source concerns the program to develop a Lie theory of semigroups. Given a closed subsemigroup $S$ of Lie group $G$, one defines the tangent object of $S$ in the Lie algebra $L(G)$ by

$$
L(S)=\{x \in L(G): \exp (t x) \in S \text { for all } t \geqq 0\}
$$

It turns out that $L(S)$ is a cone or wedge [14] $L(S)+L(S) \subseteq L(S)$ and $\mathbb{R}^{+} L(S) \subseteq L(S)$; we prefer to call these tangent objects wedges to emphasize the crucial role played by the maximal subspace $H(L(S))=L(S) \cap-L(S)$ contained in $L(S)$. The wedges turn out to be Lie wedges, i.e., wedges that are invariant under the action of $e^{a d x}$ for each $x \in H(L(S))$. Conversely given a Lie wedge, there is at least a local semigroup for which it is the tangent wedge (see [15, 16, 10 and 21] for these developments). However, unlike the group case, there need not exist global semigroups having a given Lie wedge as tangent object. As part of a program of trying to identify precisely those Lie wedges $W$ that are the tangent objects of some global semigroup, it is important to ascertain whether or not $\exp (W)$ generates all of $G$ or not.

The second source where this problem arises is in connection with certain problems concerning accessibility in non-linear geometric control theory. These problems can frequently be transferred to the question of whether the points accessible from the identity of a Lie group along a given set $A$ of invariant vector fields consists of all of $G$. Again this is the question of whether the subsemigroup generated by $\exp \left(\mathbb{R}^{+} A\right)$ is all of $G$. The problem has been considered for Lie groups which are the semidirect produce of a vector group and a compact group [1], semisimple groups [19, 7], nilpotent groups [11], and semidirect products of nilpotent and compact groups [8]. The results of this paper encompass all these cases except the semisimple. We refer to these papers for the method of interpreting and applying such results to non-linear systems.

If $A \subseteq L(G)$ generates $L(G)$ as a Lie algebra, then it is a basic result (see e.g. [25]) that the semigroup generated by $\exp \left(\mathbb{R}^{+} A\right)$ has non-empty (indeed dense) interior. We show in Section 5 that it must then lie in a closed maximal subsemigroup with interior if the semigroup is proper. Our approach is then to try to classify the closed maximal subsemigroup and their tangent wedges. Then $\exp \left(\mathbb{P}^{+} A\right)$ generates all of $G$ if and only if $A$ is not contained in the tangent set of some maximal subsemigroup. (See Section 5 for more details.) 
A distinctive feature of the approach taken here is the consideration of maximal semigroups with interior instead of maximal open subsemigroups (as in $[29,8]$ ). This allows the application of the algebraic machinery of maximal semigroups. Even this seems not to have been considered in the literature, and we include here those aspects which are germane to our purposes (see Sections 3 and 8).

Somewhat surprisingly, the theory of invariant comes into play from Section 9 onwards, with the crucial Invariant Wedge Theorem. These have been studied by Vinberg [27], Olshanskii [20, 21], and Paneitz [22, 23], the subject having its origins in the physical theory of causality and cone preserving transformations.

We should mention that there is significant contact with the work of Dobbins [5] on semigroups with group boundaries (see Section 6), although our results require an independent development. In the solvable case (the original focus of this paper) for the class of closed semigroups with non-empty interior, those that are maximal, those that are total, and those that have group boundaries all coincide (see Sections 6 and 11), and modulo a normal subgroup of the boundary fall into one of two classes.

Section 12 extends the results to the case of the extension of a solvable group by a compact group, and Section 13 suggests future lines of investigation. The reader is referred to [17] for basic Lie group theory and to [14] for basic Lie theory of semigroups.

\section{Algebraic generalities}

Throughout this paper $G$ denotes a group with identity $e$ and multiplication denoted by juxtaposition. A subset $S \subseteq G$ is a subsemigroup if $S S \subseteq S$. A submonoid is a subsemigroup which contains $e$. A non-empty subset $I$ of a subsemigroup $S$ is called a left (resp. right) ideal of $S$ if $S I \subseteq I$ (resp. $I S \subseteq S$ ). An ideal of $S$ is a subset $I$ which is both a left and right ideal.

Proposition 1.1. Let $S$ be a submonoid of $G$. Then

$$
H(S)=S \cap S^{-1}=\left\{g \in S: g^{-1} \in S\right\}
$$

is a subgroup of $G$ which contains all other subgroups in $S$. If $S \neq H(S)$, then $S^{*}=S \backslash H(S)$ is an ideal of $S$ which contains all proper left (resp. right resp. two-sided) ideals of $S$.

Proof. Since $H(S)$ is the intersection of two submonoids, it is a submonoid. It is clearly closed under inversion, so is a subgroup.

If $K \subseteq S$ is a subgroup, then $K=K \cap K^{-1} \subseteq S \cap S^{-1}=H(S)$, so $H(S)$ contains all subgroups contained in $S$.

Let $s \in S, t \in S^{\#}$ and suppose $s t \in H(S)$. Then $t^{-1} s^{-1}=(s t)^{-1} \in H(S)$. Hence $t^{-1}=$ $\left(t^{-1} s^{-1}\right) s \in S$; but this last assertion contradicts $t \notin H(S)$. Thus $s t \in S^{\#}$, i.e., $S^{\#}$ a left ideal. Similarly it is a right ideal.

If a left ideal $L$ meets $H(S)$, then $e \in H(S) L \subseteq L$, and hence $S=S e \subseteq S L \subseteq L$, i.e. $L=S$. It follows that $S^{\#}$ contains every proper left ideal.

Terminology 1.2. The subgroup $H(S)$ is called the group of units of $S$. The 
complement $S \backslash H(S)$, denoted $S^{\#}$, is called the maximal ideal of $S$. A non-empty subset $A \subseteq G$ is invariant or normal if $g \mathrm{Ag}^{-1}=A$ for all $g \in G$.

Proposition 1.3. Let $S$ be a submonoid of $G$.

(i) The largest invariant submonoid of $G$ contained in $S$ is given by

$$
S_{N}=\bigcap\left\{g S g^{-1}: g \in G\right\}
$$

(ii) The largest normal subgroup of $G$ contained in $S$ is given by

$$
\operatorname{Core}(S)=\bigcap\left\{g H(S) g^{-1}: g \in G\right\}=H\left(S_{N}\right)
$$

Proof. (i) The set $S_{N}$ is a submonoid since it is the intersection of submonoids. Since inner automorphisms distribute over intersections, $S_{N}$ is invariant. If $T$ is invariant, $T \subseteq S$, then

$$
T=\bigcap\left\{g T g^{-1}: g \in G\right\} \subseteq \bigcap\left\{g S g^{-1}: g \in G\right\}=S_{N}
$$

(ii) Core $(S)$ is the intersection of groups, hence a group. As in (i), $\operatorname{Core}(S)$ is the largest invariant set contained in $H(S)$. Since by Proposition 1.1, $H(S)$ contains every subgroup of $S$, it follows that $\operatorname{Core}(S)$ contains every normal subgroup of $S$. Since $H\left(S_{N}\right)$ is normal, $H\left(S_{N}\right) \subseteq \operatorname{Core}(S)$. But since $\operatorname{Core}(S) \subseteq S_{N}$, by Proposition $1.1 \operatorname{Core}(S) \subseteq H\left(S_{N}\right)$.

Definition 1.4. We say that a submonoid $S$ is reduced in $G$ if $\operatorname{Core}(S)=\{e\}$.

Proposition 1.5. If $S$ is a submonoid of $G$, then $S / C o r e(S)$ is reduced in $G / \operatorname{Core}(S)$.

Proof. Let $\phi: G \rightarrow G / \operatorname{Core}(S)$ be the natural homomorphism. If $K \subseteq \phi(S)$ is a normal subgroup, then $\phi^{-1}(K)$ is a normal subgroup of $G$, and $\phi^{-1}(K) \subseteq \phi^{-1} \phi(S)=$ $S \cdot \operatorname{Core}(S) \subseteq S$. Thus $\phi^{-1}(K) \subseteq \operatorname{Core}(S)$ by (ii) of Proposition 1.3. So $K=\phi \phi^{-1}(K) \subseteq$ $\phi(\operatorname{Core}(S))$, i.e., $K$ is trivial.

Definition 1.6. Let $S$ be a submonoid of $G$. The reduction of the pair $(G, S)$ is the pair $\left(G_{R}, S_{R}\right)$ where $G_{R}=G / \operatorname{Core}(S)$ and $S_{R}=S / \operatorname{Core}(S)$.

\section{Semigroups and order}

We review without proofs some elementary properties concerning the close relation between orders on $G$ and subsemigroups of $G$ (see, e.g., [6]). These will be implicit in later developments.

Given a subsemigroup $S \subseteq G$, we define a relation

$$
\leqq s \text { by } x \leqq s y \text { if } y x^{-1} \in S .
$$


This relation satisfies

(i) $x \leqq y, y \leqq z$ implies $x \leqq z$ (transitivity),

(ii) $x \leqq y$ implies $x z \leqq y z$ (right compatibility).

Furthermore, the equality $S=\{x: e \leqq s x\}$ allows one to recover $S$ as the set of positive elements.

Conversely given a transitive, right-compatible relation $\leqq$ on $G$, the set $P(\leqq)=$ $\{x \in G: e \leqq x\}$ of positive elements is a subsemigroup, and the original order $\leqq$ is equal to the induced order $\leqq p$. Thus the mutually inverse constructions of passing from $S$ to $\leqq s$ and from $\leqq$ to $P(\leqq)$ yield a bijection between subsemigroups of a group and transitive, right-compactible relations.

Proposition 2.1. Let $S$ be a subsemigroup of $G$.

(i) $e \in S$ if and only if $\leqq s$ is reflexive;

(ii) $\leqq s$ is antisymmetric if and only if $H(S)=S \cap S^{-1} \subseteq\{e\}$;

(iii) $\leqq_{s}$ is also left compatible if and only if $S$ is invariant.

Note that traditionally ordered groups are assumed to satisfy (ii). However, that will rarely be the case in our approach.

Definition 2.2. We say that $S \subseteq G$ is total if $G=S \cup S^{-1}$.

Proposition 2.3. Let $S$ be a subsemigroup of $G$. Then $S$ is total if and only if given $x, y \in G$, either $x \leqq y$ or $y \leqq x$.

\section{Maximal semigroups}

In this section we develop the algebraic machinery necessary for the later developments.

Definition 3.1. A subsemigroup $M$ of a group $G$ is called a maximal subsemigroup of $G$ if (i) the only subsemigroups containing $M$ are $M$ and $G$, and (ii) $M$ is not a subgroup. (Condition (ii) is a technical convenience, insuring the existence of the maximal ideal $M^{\#}=M \backslash H(M)$.)

Remark 3.2. If $M$ is a maximal subsemigroup of $G$, then $e \in M$ (otherwise consider $\{e\} \cup M)$.

Lemma 3.3. Let $M$ be a maximal subsemigroup of $G$, and $T$ a submonoid with $T^{-1} \nsubseteq M$. If $M T^{-1} \subseteq T^{-1} M$, then $T^{-1} M=G$.

Proof. We have $T^{-1} M T^{-1} M \subseteq T^{-1} T^{-1} M M \subseteq T^{-1} M$, so $T^{-1} M$ is a subsemigroup containing $T^{-1}$ (since $e \in M$ ) and $M$. By maximality of $M, G=T^{-1} M$.

Although elementary in nature, the next proposition is crucial in the theory of 
maximal semigroups. It is often applied by showing that $S$ cannot meet $M$ "deeply" (condition (i)), and hence $M$ "swallows" $S^{-1}$.

Proposition 3.4 (The Swallowing Lemma). Let $M$ be a maximal subsemigroup of $G$ and $S$ a subsemigroup with $M S^{-1} \subseteq S^{-1} M$ (which is the case if $S$ or $M$ is normal). Then either

(i) $S \cap I \neq \varnothing$ for every left ideal $I$ of $M$, or

(ii) $S^{-1} \subseteq M$.

Proof. Suppose $S^{-1} \Phi M$. Then we show (i) holds. Let $T=S \cup\{e\}$. Then $M T^{-1} \subseteq T^{-1} M$. By Lemma 3.3, $T^{-1} M=G$.

Let $I$ be a left ideal of $M$. Pick $x \in M^{*}$ and $y \in I$. Then $z=x y \in M^{\#} \cap I$. Since $T^{-1} M=G, z^{-1}=s^{-1} m$ for some $s \in T, m \in M$. Hence $s=m z \in M I \subseteq I$. Also $s \neq e$ since $z \in M^{\#}$ implies $z^{-1} \notin M$. Thus $s \in S$, and $S \cap I \neq \varnothing$.

Corollary 3.5. If $M$ is a maximal subsemigroup of $G$ and $H$ is a normal torsion subgroup, then $H \subseteq M$.

Proof. Suppose $H \cap M^{*} \neq \varnothing$. Let $g \in H \cap M^{\#}$. Then $\left\{g^{n}: n \geqq 1\right\} \subseteq M^{\#}$. Since $H$ is torsion, $g^{n}=e$ for some $n$. Thus $e \in M^{\#}=M \backslash H(M)$, a contradiction. Hence $H=H^{-1} \subseteq M$ by the Swallowing Lemma 3.4.

Lemma 3.6. Let $S$ be a subsemigroup of the integers $(\mathbb{Z},+)$ containing both a positive number and a negative number. Then $S$ is a subgroup of $\mathbb{Z}$.

Proof. Suppose the maximal ideal $S^{\#}=S \backslash H(S)$ is non-empty. Let $m$ be the number in $S^{\#}$ closest to 0 , and let $n \in S$ be the number closest to 0 of opposite sign. Then $m+n \in S^{\#}$ and closer to 0 than one of $m$ or $n$, a contradiction.

Proposition 3.7. Let $M$ be a maximal subsemigroup of $G$ and let $x \in G$ satisfy $x M \subseteq M x$. Then $x \in M \cup M^{-1}$.

Proof. Let $T=\left\{x^{n}: n \geqq 1\right\}$. Since $Q=\{y: y M \subseteq M y\}$ is a subsemigroup, $T \subseteq Q$. Hence $T M \subseteq M T$. Similarly $M x^{-1} \subseteq x^{-1} M$ implies $M T^{-1} \subseteq T^{-1} M$.

If $T \subseteq M$ or $T^{-1} \subseteq M$, the proof is complete. If neither of these hold, then by the Swallowing Lemma (and its dual), $T \cap M^{*} \neq \varnothing$ and $T^{-1} \cap M^{\#} \neq \varnothing$. Let $S=\left\{m \in \mathbb{Z}: x^{m} \in M^{\#}\right\}$. It is immediate that $S$ is a semigroup and $S$ contains both positive and negative numbers. By Lemma 3.6, $0 \in S$. Hence $e=x^{0} \in M^{\#}$, a contradiction. Thus this final case cannot occur.

Corollary 3.8 Let $M$ be a maximal subsemigroup of $G$. Let $Z(G)$ denote the centre of G. Then $M \cap Z(G)$ is total in $Z(G)$.

Proof. $M \cap Z(G)$ is total in $Z(G)$ if and only if $Z(G) \subseteq M \cup M^{-1}$. The latter follows from Proposition 3.7. 
Corollary 3.9 A maximal subsemigroup of an abelian group is total.

Corollary 3.10. Let $M$ be a maximal subsemigroup of $G$. If $M$ is invariant, then $M$ is total.

We consider now the interaction of maximal subsemigroups with normal abelian subgroups.

Proposition 3.11 (The Purity Lemma). Let $M$ be a maximal subsemigroup of $G$, let $H$ be a normal abelian subgroup, and let $x \in H$. If $x^{n} \in M$ for some $n>1$, then $x \in M$.

Proof. Suppose $x \notin M$. Then the subsemigroup generated by $M \cup\{x\}$ is all of $G$ (by maximality of $M$ ). Note that since $1 \in M$, any member of $\mathrm{G}$ is either in $M$ or has a representation $m_{1} x m_{2} x \ldots m_{k-1} x m_{k}$ (since such products together with $M$ form a semigroup containing $x$ and $M$ ). Let $g \in M^{*} \cap H$ (the Swallowing Lemma 3.4). Then for some $m_{1}, \ldots, m_{k} \in M$,

$$
g^{-1}=m_{1} x \ldots x m_{k}=\left(m_{1} \ldots m_{k}\right) x^{m_{2} \ldots m_{k}} \ldots x^{m_{k}}=m y
$$

(where $z^{w}=w^{-1} z w$ and $m=m_{1} \ldots m_{k}$ ). Since $H$ is normal and $x \in H$, we conclude $y \in H$. Thus $m=g^{-1} y^{-1} \in H$. So $m$ commutes with $y$. Hence

$$
g^{-n}=m^{n} y^{n}=m^{n-1} m\left(x^{n}\right)^{m_{2} \ldots m_{k}} \ldots\left(x^{n}\right)^{m_{k}}=m^{n-1} m_{1} x^{n} m_{2} x^{n} \ldots x^{n} m_{k} \in M
$$

since $x^{n} \in M$. But $g \in M^{\#}$ implies $g^{n} \in M^{\#}$. Therefore $e=g^{n} g^{-n} \in M^{\#} M \subseteq M^{\#}$, a contradiction.

We close this section with an elementary, but useful, lemma.

Lemma 3.12 (The Reduction Lemma). Let $\phi: G \rightarrow H$ be a homomorphism onto $H$, and let $S$ be a submonoid of $H$. Then $S$ is maximal (resp. total resp. invariant) in $H$ if and only if $\phi^{-1}(S)$ is maximal (resp. total resp. invariant) in $G$.

Proof. Suppose $S$ is maximal in $H$. Then $\phi^{-1}(S)$ is not a group since $S$ is not a group. If $T$ is a subsemigroup containing $\phi^{-1}(S)$, then $T$ contains the kernel of $\phi$, so $T=\phi^{-1}(\phi(T))$. Since $S \subseteq \phi(T), \phi(T)=S$ or $\phi(T)=H$. Thus $T=\phi^{-1} \phi(T)=\phi^{-1}(S)$ or $T=\phi^{-1} \phi(T)=\phi^{-1}(H)=G$. Hence $\phi^{-1}(S)$ is maximal.

The remaining arguments are all similarly straightforward.

Note that since Core $(S) \subseteq S$, we have $S=\phi^{-1}(\phi(S))=\phi^{-1}\left(S_{R}\right)$, where $\phi: G \rightarrow G_{R}$.

Corollary 3.13. Let $S$ be a submonoid in $G$. Then $S$ is maximal in $G$ if and only if $S_{R}$ is maximal in $G_{R}=G / \operatorname{Core}(S)$.

Corollary 3.13 shows that all maximal subsemigroups arise as inverse images of reduced ones. It is then the latter that we seek to characterize. 


\section{Reversibility}

Although the material of this section is not needed later, it appears worthwhile to amplify some ideas implicit in the preceding section.

Definition 4.1. For non-empty $A, B \subseteq G$, the pair $(A, B)$ is called a right-reversing pair if $A b \cap B a \neq \varnothing$ for all $a \in A, b \in B$. A subsemigroup $S$ of $G$ is called right-reversible if $S x \cap S y \neq \varnothing$ for all $x, y \in S$.

Note that $(A, B)$ is a right-reversing pair if and only if $(B, A)$ is.

The following lemma gives frequently used conditions for a pair to be right-reversing (cf. Lemma 3.3 and Proposition 3.4).

Lemma 4.2. Let $A, B \subseteq G$ be non-empty. Then $(A, B)$ is right-reversing if and only if $B A^{-1} \subseteq A^{-1} B$. This happens if $A$ or $B$ is invariant.

Proof. Suppose $(A, B)$ is right-reversing. Then for $a \in A, b \in B$, there exist $u \in A, v \in B$ such that $u b=v a$. Then $b a^{-1}=u^{-1} v \in A^{-1} B$. Thus $B A^{-1} \subseteq A^{-1} B$. The equivalence follows since the steps are reversible.

If $A$ is invariant, $A B^{-1}=B^{-1} A$. Taking inverse we obtain $B A^{-1}=A^{-1} B$.

The process of embedding the natural numbers into the integers extends to rightreversible cancellable semigroups. Indeed a semigroup $S$ embeds isomorphically in a group of left quotients (i.e. $S^{-1} S=G$ ) if and only if $S$ is cancellable and right-reversible. These are called Ore's conditions (see Section 1.10 of [4]). Note that Lemma 3.3 realizes $G$ as a certain set of left quotients.

Proposition 4.3. Let $M$ be a maximal subsemigroup of $G$. Then either

(i) $G_{R}$ contains no non-trivial normal abelian subgroup, or

(ii) $M$ is right-reversible, and $M^{-1} M=G$.

Proof. Suppose $G_{R}$ contains a normal abelian subgroup $H \neq\{e\}$. Let $x, y \in M_{R}$. Since $H \nsubseteq M_{R}$ (otherwise $M_{R}$ is not reduced in $G_{R}$ ), by the Swallowing Lemma $H \cap M_{R} x \neq \varnothing$ and $H \cap M_{R} y \neq \varnothing$ (since by Lemma $3.12 M_{R}$ is maximal). Let $h_{1}=m_{1} x \in H \cap M_{R} x$ and let $h_{2}=m_{2} y \in H \cap M_{R} y$. Then $h_{1} h_{2}=m_{1} x m_{2} y \in M_{R} y$ and $h_{2} h_{1}=m_{2} y m_{1} x \in M_{R} x$. Since $h_{1} h_{2}=h_{2} h_{1}, M_{R} x \cap M_{R} y \neq \varnothing$. Thus $M_{R}$ is right-reversible.

Now let $a, b \in M$. Then $M_{R} \vec{a} \cap M_{R} \bar{b} \neq \varnothing$, where $\bar{a}$ resp. $\bar{b}$ is the image of $a$ resp. $b$ in $G_{R}$. So $g m_{1} a=m_{2} b$ for some $g \in \operatorname{Core}(M), m_{1}, m_{2} \in M$. Thus $M a \cap M b \neq \varnothing$. Hence $M$ is right-reversible. By Lemmas 3.3 and $4.2, M^{-1} M=G$.

Corollary 4.4. Let $M$ be a maximal subsemigroup of a solvable group $G$. Then $M$ is right-reversible and $G=M^{-1} M$.

Proof. Since $G$ is solvable, so is $G_{R}$. Thus $G_{R}$ has a non-trivial normal abelian subgroup. The corollary follows from Proposition 4.3. 


\section{Topological generalities}

Throughout this section $G$ denotes a locally generated topological group (i.e., $G$ is the only subgroup containing a neighborhood of the identity $e$ ). We recall the elementary fact that every connected topological group is locally generated.

Lemma 5.1. Let $U \neq \varnothing$ be an open set, $U \subseteq S$, where $S$ is a proper subsemigroup of $G$. Then $S \cap U^{-1}=\varnothing$.

Proof. Suppose $s \in S \cap U^{-1}$. Then $s^{-1} \in U \subseteq S$, and thus $e=s s^{-1} \in s U \subseteq s S \subseteq S$. Thus $S$ contains the open neighborhood $s U$ of $e$, and hence $S=G$, contradiction.

Proposition 5.2. Let $S$ be a proper (open) subsemigroup of $G$ with $\operatorname{int}(S) \neq \varnothing$. Then $S$ is contained in a maximal (open) subsemigroup.

Proof. Let $\mathscr{M}$ be a maximal tower of proper (open) subsemigroups of $G$ containing $S$, and let $M$ be their union. If $U=\operatorname{int}(S)$, then by Lemma $4.1 T \cap U^{-1}=\varnothing$ for all $T \in \mathscr{M}$. Hence $M \cap U^{-1}=\varnothing$, so $M$ is proper and also not a group. Clearly $M$ is a maximal (open) subsemigroup.

We pause to consider the aims of this paper. A central purpose is to develop criteria that allow one to determine whether the subsemigroup generated by an arbitrary subset $B$ of $G$ is all of $G$ or not. Proposition 5.2 points to our approach.

Corollary 5.3. Suppose $B \subseteq G$ has the property that the semigroup it generates has nonempty interior in $G$. If $B$ is not contained in a maximal subsemigroup with non-empty interior, then the subsemigroup generated by $B$ is all of $G$.

Thus if we can specify the maximal subsemigroups with non-empty interior, we need only check whether $B$ is a subset of one of these maximal subsemigroups.

We remark also that the approach adopted here differs slightly (but significantly) from most earlier approaches, where maximal open subsemigroups were considered in the topological setting. Our approach allows the use of the machinery of Section 3, since we work with genuinely maximal subsemigroups.

Proposition 5.4. If $M$ is a maximal subsemigroup and int $M \neq \varnothing$, then $M$ is closed.

Proof. Let $U=$ int $M$. By Lemma $5.1 M \cap U^{-1}=\varnothing$, so $\bar{M} \cap U^{-1}=\varnothing$. Thus $\bar{M}$ is a proper subsemigroup containing $M$, so by maximality, $M=\bar{M}$.

Proposition 5.5. If $S$ is a closed subsemigroup of $G$, then $H(S)$ and $\operatorname{Core}(S)$ are closed.

Proof. Core $(S)$ is the intersection of all $g H(S) g^{-1}$ (see Proposition 1.3) and hence closed, since $H(S)=S \cap S^{-1}$ is closed.

Remark 5.6. If $S$ is a subsemigroup of $G$ with $\operatorname{int}(S) \neq \varnothing$, then $\operatorname{int}(S)$ is an ideal of $S$ (by openness of translation). 
Proposition 5.7. Let $M$ be a maximal subsemigroup of $G$ with $\operatorname{int}(M) \neq \varnothing$. If $H$ is a compact subgroup, then $H \cap \operatorname{int}(M)=\varnothing$. If $H$ is compact and normal, then $H \subseteq \operatorname{Core}(M)$.

Proof. Suppose $H \cap \operatorname{int}(M) \neq \varnothing$. Since $H \cap \operatorname{int}(M)$ is an open subsemigroup of the compact group $H$, it is a compact open subgroup (see e.g. [28]). Hence $1 \in \operatorname{int} M$, so $M=G$, a contradiction. Then by the Swallowing Lemma $H \subseteq M$ if $H$ is normal. It follows that $H \subseteq \operatorname{Core}(M)$ in this case, since $\operatorname{Core}(M)$ is the largest normal subgroup of $M$.

Proposition 5.8. If $M$ is a maximal subsemigroup of $G$ and $H(M) \cap(\text { int } M)^{-} \neq \varnothing$ (in particular, if $\left.e \in(\text { int } M)^{-}\right)$, then int $M$ is a maximal open subsemigroup and $M=(\text { int } M)^{-}$.

Proof. Let $I=$ int $M$. By Remark 5.6, $I$ is an ideal of $M$, so $I$ is an open subsemigroup. Then $\bar{I} \subseteq \bar{M}=M$ (Proposition 5.4). Conversely if $g \in H(M) \cap(\text { int } M)^{-}$, then $M=M g \subseteq M \bar{I} \subseteq \overline{M I} \subseteq \bar{I}$.

If $T \neq G$ is an open subsemigroup containing $I$, then $M=\bar{I} \subseteq \bar{T}$ and $\bar{T} \neq G$ (Lemma 5.1) imply $M=\bar{T}$. Thus $T \subseteq$ int $\bar{T}=$ int $M$. Thus int $M$ is a maximal open subsemigroup.

\section{Total semigroups}

Corollaries 3.9 and 3.10 gave sufficient conditions for a maximal subsemigroup to be total. In this section we consider this situation in more detail and relate it to known results. Throughout $G$ denotes a locally generated topological group.

Proposition 6.1. Let $M$ be a closed total subsemigroup of $G$. Then $M$ is maximal.

Proof. Suppose $x \notin M$. Let $U=G \backslash M$. Then $U$ is open, and $U \subseteq M^{-1}$ (since $G=$ $\left.M \cup M^{-1}\right)$. Thus $U^{-1} \subseteq M$. The subsemigroup $T$ generated by $\{x\} \cup M$ contains $x U^{-1}$, an open set containing $e$. Thus $T=G$ (by local generation).

The next proposition derives basic properties of such semigroups.

Proposition 6.2. Let $M$ be a closed subsemigroup of $G$. Then $M$ is total iff $G=M^{\#} \cup H(M) \cup\left(M^{\#}\right)^{-1}$. In this case $M^{\#}=$ int $M, M$ is the closure of $M^{\#}$, and $H(M)=\operatorname{Bd}\left(M^{\#}\right)\left(=\left(M^{\#}\right)^{-} \backslash\right.$ int $\left.M^{\#}\right)$.

Proof. The equivalence follows from the equality

$$
M \cup M^{-1}=M^{\#} \cup H(M) \cup H(M)^{-1} \cup\left(M^{\#}\right)^{-1}=M^{\#} \cup H(M) \cup\left(M^{\#}\right)^{-1} .
$$

If $M$ is total, then since $M=M^{\#} \cup H(M)$ and $M \cap\left(M^{\#}\right)^{-1}=\varnothing,\left(M^{\#}\right)^{-1}=G \backslash M$ is open, and hence $M^{\#}$ is open. Thus $M^{\#} \subseteq$ int $M$. Since int $M$ is an ideal (Remark 5.6) and $M^{\#}$ is the largest ideal (Proposition 1.1), int $M \subseteq M^{\#}$. Hence $M^{\#}=$ int $M$.

If $M^{\#}=\left(M^{\#}\right)^{-}$, then $\left(M^{\#}\right)^{-1}$ is also closed, so $H(M)$ is open. But then $G$ is not locally generated. Hence there exists $g \in H(M) \cap\left(M^{\#}\right)^{-}$. Then by Proposition 5.8 $M=\left(M^{\#}\right)^{-}$. Thus $\operatorname{Bd}\left(M^{\#}\right)=M \backslash M^{\#}=H(M)$. 
Closed total subsemigroups are maximal, but the converse does not hold in general. We are interested in determining sufficient conditions to insure the converse. We begin with two important special examples where we can calculate all closed maximal subsemigroups.

Proposition 6.3. Let $M$ be a maximal closed subsemigroup of $(\mathbb{R},+)$. Then either $M=\mathbb{R}^{+}=\{x \in \mathbb{R}: x \geqq 0\}$ or $M=-\mathbb{R}^{+}$.

Proof. Let $y \in M, y>0$. By the Purity Lemma (3.11), $(1 / n) y \in M$ for all positive integers $n$. Thus $(m / n) y \in M$ for all positive rationals. Since $M$ is closed, $\mathbb{R}^{+} \subseteq M$. Since $\mathbb{R}^{+}$is closed and total, by Proposition 6.1 it is maximal. Hence $\mathbb{R}^{+}=M$. Similarly if $M$ contains a negative number, $M=-\mathbb{B}^{+}$.

Note that the preceding argument could be applied to any subgroup of $\mathbb{R}$ that was $n$-divisible for some $n$ (e.g., the rationals).

We next determine the closed reduced maximal subsemigroups of the unique two dimensional non-abelian connected Lie group. This group may be thought of as the component of the identity in the group of affine motions on $\mathbb{R}$; hence we denote it $\mathrm{Aff}^{+}$. We show that the closed maximal subsemigroups are the half-spaces with boundary some one-dimensional subgroup. These are all isomorphic to $\mathrm{Aff}^{++}$, the semigroup of affine motions with translation term non-negative.

Alternately $\mathrm{Aff}^{+}$may be identified with the multiplicative matrix group $\left\{\left[\begin{array}{ll}x & y \\ 0 & 1\end{array}\right]: x>0\right\}$, or with order pairs $\{(x, y): x>0\}$ with multiplication $(u, v)(x, y)=(u x, u y+v)$. Aff ${ }^{++}$then has $y \geqq 0$.

Lemma 6.4. Let $G$ be the group of positive reals under multiplication. Given $s, t \in G$ with $0<s<1<t, \varepsilon>0$, and a positive integer $N$, there exists positive integers $j, k$ with $j \geqq N$ such that $\left|s^{j} t^{k}-1\right|<\varepsilon$.

Proof. Consider first the additive group $\mathbb{R}$ and positive real numbers $x$ and $y$. The set $\{n x: n \geqq 1\}$ is a cyclic semigroup in the compact group $\mathbb{R} / \mathbb{Z} y$. Hence its closure is a compact semigroup, and thus a compact group. So the elements $n x$ cluster to the identity of $\mathbb{R} / \mathbb{Z} y$. Hence there exists $j \geqq N$ such that $|j x-k y|<\varepsilon$ for some $k>0$, i.e. $|j(-x)+k y|<\varepsilon$. The lemma now follows from this derivation by applying the exponential function from the additive reals to the multiplicative positive reals.

Proposition 6.5. Let $\mathrm{G}=\mathrm{Aff}^{+}$be the (unique) Lie group with Lie algebra the twodimensional non-abelian Lie algebra. If a closed semigroup $M$ is maximal, then there exists a one-dimensional group such that $M$ is the union of this group and one of the two components of its complement. In particular, $M$ is total.

Proof. We identify $G$ as $\{(x, y): x>0, y \in \mathbb{R}\}$ with multiplication $(u, v)(x, y)=$ $(u x, u y+v)$. Then $G$ has identity $e=(1,0)$ and the one-dimensional groups are the straight lines through $e$, i.e., all $(x, y)$ such that $y=m x-m$ for some fixed $m$. The vertical line $H=\{(1, y): y \in \mathbb{R}\}$ is the only non-trivial normal subgroup. The sets $H^{+}=\{(1, y): y \geqq 0\}$ and $H^{-}=\{(1, y): y \leqq 0\}$ are invariant subsemigroups. 
By the Swallowing Lemma either $H \subseteq M$ or $M^{\#} \cap H \neq \varnothing$. Suppose $H \subseteq M$. Then $G / H$ is isomorphic to the multiplicative positive reals, which in turn is isomorphic to $(\mathbb{R},+)$, and $M / H$ is a closed maximal subsemigroup. By Proposition $6.3 M / H$ corresponds either to $(0,1]$ or $[1, \infty)$. Then $M=\{(x, y): 0<x \leqq 1\}$ or $M=\{(x, y): 1 \leqq x\}$. In either case $M$ is a "half-space" of the desired type with boundary $H$.

The more complicated case is $M^{\#} \cap H \neq \varnothing$. Let us assume $(1, y) \in M^{\#}$ for some $y>0$. By the Purity Lemma (3.11) $(1,(1 / n) y) \in M$ for all positive $n,(1,(m / n) y) \in M$ by the semigroup property for $m / n>0$, and so $H^{+} \subseteq M$ since $M$ is closed. Since $H^{+}$is total in $H$ and hence maximal and since $H \nsubseteq M$ (since $M^{\#} \cap H \neq \varnothing$ ), then $M \cap H=H^{+}$.

Since $H^{-}$is normal, $H^{-} M$ is a subsemigroup properly containing $M$. Hence $H^{-} M=G$. Since multiplying any element of $G$ on the left by $(1, y)$ shifts the element $y$ units vertically, it must be the case that $(\{x\} \times \mathbb{R}) \cap M \neq \varnothing$ for all $x>0$.

Let $(s, m s-m),(t, \mu t-\mu) \in M$ with $0<s<1<t$. Using the fact that the straight lines through $(1,0)$ are subgroups or by direct computation, one obtains that the powers of these elements are given by $\left(s^{j}, m s^{j}-m\right)$ and $\left(t^{k}, \mu t^{k}-\mu\right)$. The product is again in $M$ and is given by

$$
\left(s^{j} t^{k}, \mu s^{j} t^{k}-\mu s^{j}+m s^{j}-m\right)=\left(s^{j} t^{k}, \mu\left(s^{j} t^{k}-1\right)+(m-\mu)\left(s^{j}-1\right)\right)
$$

If $j, k$ are chosen as in Lemma 6.4 , we see that this product can be made arbitrarily close to $(1, \mu-m)$, which must thus be a member of $M$. Hence $\mu-m \geqq 0$, i.e. $m \leqq \mu$.

From the preceding paragraph it follows that

$$
a=\sup \{m:(s, m s-m) \in M \text { for some } s<1\} \leqq \inf \{\mu:(t, \mu t-\mu) \in M \text { for some } t>1\}=b
$$

If $d$ is chosen so that $a \leqq d \leqq b$, then it follows that the region above the straight line of slope $d$ through $(1,0)$ contains $M$.

Note that in Proposition 6.2 the open semigroup $M^{\#}$ has boundary a subgroup. This situation has been explored in the literature and we mention a highly non-trivial converse that has been established by Dobbins [5] with important contributions by that paper's referee, Hofmann, and the finishing touches added by Poguntke [24]. Unfortunately the results are not directly applicable to maximal semigroups. Although we derive analogous results, the techniques are quite different.

Theorem 6.6. Let $S$ be a proper open subsemigroup of a locally compact connected group $G$ such that $\mathrm{Bd}(S)$ is a group. Then $\bar{S}$ is total in $G$, and $\left(G_{R}, \bar{S}_{R}\right)$ falls into one of the following three cases up to topological isomorphism:

(i) $G_{R}=\mathbb{R}, \bar{S}_{R}=\mathbb{R}^{+}$;

(ii) $G_{R}=$ Aff $^{+}, \bar{S}_{R}=$ Aff $^{++}=\left\{(x, y) \in\right.$ Aff $\left.^{+}: y \geqq 0\right\}$;

(iii) $G_{R}=\tilde{S} L(Z, \mathbb{R})$, the simply connected covering groups of $S L(2, \mathbb{R}), \bar{S}_{R}=$ the closure of one of the components of $\tilde{S} L\left(2, \mathbb{R} \backslash \exp (H)\right.$, where $H=\left\{\left[\begin{array}{cc}x & y \\ 0 & -x\end{array}\right]: x, y \in \mathbb{R}\right\}$ and exp:sl $(2, \mathbb{R}) \rightarrow \widetilde{S} L(2, \mathbb{R})$ is the exponential mapping for $\tilde{S} L(2, \mathbb{R})$. 
In light of Proposition 6.2 and Theorem 6.6 the closed total subsemigroups can be characterized as extensions of groups by one of the three semigroups of Theorem 6.6. (It would be of interest to know whether there was a more direct proof that an open semigroup with a group boundary has closure which is total.) Note that $\tilde{S} L(2, \mathbb{R})$ is the only simple Lie group admitting total subsemigroups. Further information on maximal semigroups in $S L(2, \mathbb{R})$ and $\widetilde{S} L(2, \mathbb{R})$ may be found in [9].

Note that case (i) of Theorem 6.6 is the only reduced case where the semigroup is invariant. We show later that maximal subsemigroups are also total in the solvable group case and give rise to case (i) or case (ii).

Finally we remark that Theorems 6.2 and 6.6 relate to a theorem of Sophus Lie himself. Let $G$ be a connected Lie group with a closed total subsemigroup $S$. Then a total order is induced on $G / H(S), G / H(S)$ is order and topological isomorphic to the reals (indeed this can easily be deduced from Theorem 6.6 since Core $S \subseteq H(S)$ ), and $G$ acts on $G / H(S)$. This induces an infinitesimal action of $L(G)$ on $G / H(S) \simeq \mathbb{R}$, and one of Lie's theorems was that the only such actions that were "effective" were ones by $L(\mathbb{R})=\mathbb{R}, L\left(\mathrm{Aff}^{+}\right)$, and $\mathrm{sl}(2)$.

\section{Invariant maximal semigroups}

We recall a useful lemma (Lemma 3.9 from [5]).

Lemma 7.1. Let $G$ be a connected locally compact group and $H$ a closed normal subgroup. If $G / H$ is simply connected, then $H$ is connected.

The following theorem overlaps with case (i) of Theorem 6.6 and much of the locally compact case could be derived from it. It also parallels results in Section 1 of [30]. Here it follows directly from the results of previous sections.

Theorem 7.2. Let $G$ be a connected topological group which is either locally compact or locally connected, and let $M$ be a closed subsemigroup. The following are equivalent:

(1) $M$ is maximal and invariant;

(2) $M$ is total and invariant;

(3) $M$ is total and $H(M)=\operatorname{Core}(M)$, i.e., $H(M)$ is normal;

(4) $M$ is maximal and $G_{R}$ is topologically isomorphic to the additive reals. (In this case the topological isomorphism must carry $M_{R}$ to $\mathbb{R}^{+}$or $-\mathbb{R}^{+}$.)

Furthermore, in the locally compact case these conditions imply $H(M)$ is connected.

Proof. The equivalence of (1) and (2) follows from Proposition 6.1 and Corollary 3.10. If $M$ is invariant, then $H(M)=M \cap M^{-1}$ is normal, and thus $H(M)=\operatorname{Core}(M)$ (see Proposition 1.3). Thus (2) implies (3).

We show (3) implies (4). In this case $G_{R}=G / H(M)$ is a totally ordered (see Propositions 2.1 and 2.3) connected topological group which is locally compact or locally connected (since the quotient mapping is open) and with a closed set $M_{R}$ of elements greater than or equal to 0 . Note the $G_{R} \backslash\{e\}$ is the disjoint union of the 
relatively closed sets $M_{R} \backslash\{e\}$ and $\left(M_{R}\right)^{-1} \backslash\{e\}$, and is hence not connected. By Exercise 4 of Chapter V, $\S 3$ of Bourbaki's Topologie Generale [2], $G_{R}$ is abelian and topologically isomorphic to $(\mathbb{R},+)$. The image of $M_{R}$ in $\mathbb{R}$ is a closed maximal subsemigroup, hence equal to $\mathbb{R}^{+}$or $-\mathbb{R}^{+}$(Proposition 6.3). This completes the proof of (4) and the parenthetical remark.

If (4) holds, then we have just seen the parenthetical remark holds. Thus $M_{R}$ is total and invariant. It follows that $M$ is total and invariant (see the Reduction Lemma 3.12). Thus (2) holds.

That $H(M)$ is also connected in the locally compact case follows from Lemma 7.1 .

Note that for topological vector spaces one obtains a type of geometric Hahn-Banach Theorem. If $K$ is a convex body with interior missing 0 , then the rays through $K$ form an additive semigroup. Extending this semigroup to a maximal one (Proposition 5.2) and forming the reduction gives a continuous linear functional into $\mathbb{R}$ (by the preceding proposition) carrying the interior of the convex set into the positive reals. For a whole theory of maximal subsemigroups in the setting of abelian topological groups together with several such applications, see [29].

\section{Nilpotent groups}

In a group $G$ denote by $[g, h]$ the commutator $g^{-1} h^{-1} g h$. If $H$ is a subgroup of $G$, let $[G, H]$ denote the subgroup generated by the set $\{[g, h]: g \in G, h \in H\}$. Define recursively $G_{n}$ by $G_{0}=G, G_{n+1}=\left[G, G_{n}\right]$. The group $G$ is nilpotent if $G_{n+1}=\{e\}$ for some $n$. Note that if $G_{n+1}=\{e\}$, then $G_{n}$ is contained in the centre of $G$.

Lemma 8.1. Suppose $G$ is a group, $M$ is a maximal subsemigroup of $G$, and $g, h \in G$ are such that $[g, h]$ is in the centre of $G$. Then $[g, h],[g, h]^{-1} \in M$.

Proof. Let $w=[g, h]$. If $w=e$, then the lemma is trivial (see Remark 3.2). By Corollary $3.8 w \in M$ or $w^{-1} \in M$. We suppose (without loss of generality) that $w \in M$ and show that also $w^{-1} \in M$.

Suppose on the contrary that $w^{-1} \notin M$. Let $S=\left\{w^{-n}: n \geqq 0\right\}$. Then $S M$ is a semigroup containing $M$ and $w^{-1}$; by maximality of $M, S M=G$. Thus $g=w^{-n} u, h=w^{-m} v$, $g^{-1}=w^{k} x, h^{-1}=w^{-p} y$ for some $n, m, k, p \geqq 0$ and $u, v, x, y \in M$. Thus $w^{n} g=u \in M$ and similarly $w^{m} h, w^{k} g^{-1}, w^{p} h^{-1} \in M$. Thus $w^{q} g, w^{q} h, w^{q} g^{-1}, w^{q} h^{-1} \in M$ where $q=n+m+k+p$ since $w \in M$ by assumption.

Now $g h=h g[g, h]$. Since $[g, h]$ is central, an easy induction yields for any $n>0$ that $g^{n} h^{n}=h^{n} g^{n}[g, h]^{n^{2}}$, i.e. $g^{n} h^{n}[g, h]^{-n^{2}}=h^{n} g^{n}$. Then for $z=w^{q}$, we have $\left(z g^{-1}\right)^{n}(z h)^{n}(z g)^{n}\left(z h^{-1}\right)^{n} \in M$ for all $n \geqq 1$ and

$$
\begin{aligned}
\left(z g^{-1}\right)^{n}(z h)^{n}(z g)^{n}\left(z h^{-1}\right)^{n} & =z^{4 n} g^{-n}\left(h^{n} g^{n}\right) h^{-n}=z^{4 n} g^{-n}\left(g^{n} h^{n}[g, h]^{-n^{2}}\right) h^{-n} \\
& =z^{4 n} w^{-n^{2}}=w^{4 n q} w^{-n^{2}}=w^{4 n q-n^{2}}
\end{aligned}
$$

Since for large $n, 4 n q-n^{2}<0$ and since $w \in M$, we conclude $w^{-1} \in M$, a contradiction. This completes the proof. 
Proposition 8.2. Let $M$ be a maximal subsemigroup of $G$ which is reduced in $G$. Then $G / Z(G)$ has trivial centre.

Proof. Suppose not. Let $\phi$ be the natural homomorphism from $G$ to $G / Z(G)$. Then there exists $g \in G$ such that $\phi(g)$ is in the centre of $G / Z(G)$, but $\phi(g)$ is not the identity. Since $g \notin Z(G)$, there exists $h \in G$ such that $g h \neq h g$. Then $g^{-1} h^{-1} g h \neq e$. But $\phi\left(g^{-1} h^{-1} g h\right)=\phi\left(g^{-1}\right) \phi\left(h^{-1}\right) \phi(g) \phi(h)=\phi\left(g^{-1}\right) \phi(g) \phi\left(h^{-1}\right) \phi(h)=\phi(e)$. Thus $[g, h] \in Z(G)$. By Lemma $8.1[g, h],[g, h]^{-1} \in M$. Since $[g, h] \in Z(G)$, the subgroup it generates is normal. But this contradicts the assumption that $M$ is reduced in $G$.

Theorem 8.3. Let $M$ be a maximal subsemigroup of a nilpotent group $G$. Then $M$ is total and invariant in $G$ and $[G, G] \subseteq H(M)$. Hence $G_{R}=G / H(M)$ is abelian and totally ordered.

Proof. Let $\left(G_{R}, M_{R}\right)$ be the reduction of $(G, M)$. By the Reduction Lemma (3.12) $M_{R}$ is maximal in $G_{R}$. Since the quotient of a nilpotent group is nilpotent, any quotient of $G_{R}$ is nilpotent, hence has non-trivial centre if it is non-trivial. By Proposition 8.2 it follows that $G_{R}$ must be abelian. Thus $[G, G] \subseteq \operatorname{Core}(M)$. By Corollary $3.9 M_{R}$ is total, and by the Reduction Lemma $M$ is total. Since $[G, G] \subseteq H(M) \subseteq M, M$ and $H(M)$ are invariant (since $g^{-1} m g m^{-1}, m \in M$ imply $g^{-1} m g \in M$ ). Thus Core $(M)=H(M)$.

Corollary 8.4. Let $G$ be a connected, nilpotent topological group which is locally compact or locally connected. Let $M$ be a maximal subsemigroup with int $M \neq \varnothing$. Then $\left(G_{R}, M_{R}\right)$ is topologically isomorphic to $\left(\mathbb{R}, \mathbb{R}^{+}\right)$. Hence $\operatorname{Core}(M)=H(M)$ is a closed normal subgroup, which is also connected in the locally compact case.

Proof. By Theorem $8.3 M$ is total and invariant, and by Proposition $5.4 M$ is closed. The rest now follows from Theorem 7.2.

Corollary 8.4 may be viewed as an alternate statement of one of the main results of [11], when it is re-interpreted at the Lie algebra level for nilpotent Lie groups.

Proposition 8.5. Let $H$ be a locally generated normal nilpotent subgroup of a locally generated topological group $G$ and let $M$ be a maximal subsemigroup with int $M \neq \varnothing$. Then $[H, H] \subseteq \operatorname{Core}(M)$.

Proof. We first show $[H, H] \cap$ int $M=\varnothing$. Suppose not. Since $e \notin$ int $M$ (otherwise $M=G$ ), int $M \cap H$ is a proper subsemigroup of $H$ with interior in $H$. By Proposition 5.2 there exists a maximal subsemigroup $S$ of $H$ containing int $M \cap H$. By Theorem 8.3 $[H, H] \subseteq S$. If $g \in[H, H] \cap$ int $M$, then $g^{-1} \in[H, H] \cap(\text { int } M)^{-1} \subseteq S \cap(\operatorname{int} M)^{-1}$; this contradicts Lemma 5.1 applied to $H$ (with $U=\operatorname{int} M \cap H$ ). Thus $[H, H] \cap$ int $M=\varnothing$.

By Remark 5.6 and the Swallowing Lemma $[H, H] \subseteq M$ (since $H$ normal implies $[H, H]$ is normal). Thus $[H, H] \subseteq \operatorname{Core}(M)$.

Corollary 8.6. Let $G$ be a locally generated nilpotent group. If $A \subseteq G$ and int $A \cap[G, G] \neq \varnothing$, then the subsemigroup generated by $A$ is all of $G$. 
Proof. Let $S$ be the subsemigroup generated by $A$. If $S \neq G$, then $S$ is contained in a maximal semigroup $M$ by Proposition 5.2. By Proposition $8.5[G, G] \subseteq \operatorname{Core}(M)$. But by hypothesis $\varnothing \neq$ int $A \cap[G, G] \subseteq$ int $M \cap[G, G]$, a contradiction since $H(M) \cap$ int $M=\varnothing$. Thus $S=G$.

Note that in Corollary 8.6 we have used the techniques of maximal semigroups to reach conclusions about which subsets of a group are generating.

\section{Invariant wedges}

Throughout this section $G$ denotes a connected Lie group with Lie algebra $L(G)$. For $g \in G$, the inner automorphism $I_{g}: G \rightarrow G$ defined by $h \rightarrow g h g^{-1}$ induces an automorphism Ad $g: L(G) \rightarrow L(G)$ such that the following square commutes:

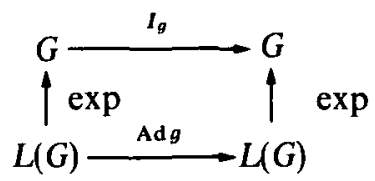

Thus $\exp (\operatorname{Ad} g(x))=g \exp (x) g^{-1}$. We recall also the basic property that if ad $x: L(G) \rightarrow L(G)$ is defined by $y \rightarrow[x, y]$, then

$$
\operatorname{Ad} \exp (x)=e^{\operatorname{ad} x}\left(=1+\operatorname{ad} x+\frac{1}{2 !}(\operatorname{ad} x)^{2}+\cdots\right) .
$$

It follows that the subgroup generated by the image of an ideal is normal and that a subalgebra of $L(G)$ is invariant under all $\mathrm{Ad} g$ if and only if it is an ideal.

Theorem 9.1. (The Invariant Wedge Theorem). Let $G$ be a connected Lie group, let exp: $L(G) \rightarrow G$ be the exponential mapping, and let $I$ be an abelian ideal of $L(G)$. If $M$ is a maximal subsemigroup of $G$ with $\operatorname{int}(M) \neq \varnothing$, then $W=\{x \in I: \exp (x) \in M\}$ is a closed wedge in $L(G)$ which is invariant under the adjoint action of $G$, and $W-W=I$.

Proof. Since $I$ is an abelian ideal, $\exp (I)$ is a normal subgroup. If $\exp (I) \subseteq M$, we are finished, since $I$ is an ideal, hence invariant under the adjoint action.

If $\exp (I) \Phi M$, then by the Swallowing Lemma, $\exp (I) \cap \operatorname{int}(M) \neq \varnothing$. Pick $h=\exp (y)$, $h \in \operatorname{int}(M), y \in I$.

Since $M$ is closed (Proposition 5.4) and exp is continuous, $W=\exp ^{-1}(M) \cap I$ is closed. Since $M$ is a subsemigroup and exp restricted to $I$ is a group homomorphism (since $I$ is abelian), $W$ is closed under addition. By the Purity Lemma (3.11) applied to $H=\exp I$, we conclude that $W$ is closed under scalar multiplication by $1 / n$. Hence $W$ is closed under scalar multiplication by positive rationals. Thus $\mathbb{R}^{+} \cdot W \subseteq \bar{W}=W$; it follows that $W$ is a wedge. Since $\exp (y) \in \operatorname{int}(M)$, there exists a neighborhood of $y$ mapping into $M$. Thus the wedge $W$ has interior in $I$ and $y$ is an interior point.

Let $x$ be any other interior point. Then $n x=y+z_{n}$ for some $z_{n} \in W$ for all sufficiently 
large $n$. (To see this, let $U$ be open in $I, x \in U \subseteq W$. Then $(1 / n) y \in x-U$ for all large $n$. Thus $(1 / n) y+u_{n}=x$, i.e. $y+z_{n}=n x$ where $z_{n}=n u_{n} \in W$.)

Since $h \in \operatorname{int}(M)$, there exists an open set $N=N^{-1}, e \in N$, with $N h \subseteq \operatorname{int}(M)$. Let $g \in N$ and let $x$ be an interior point of $W$. For large $n$, pick $z_{n} \in W$ such that $n x=y+z_{n}$. Let $a=\exp (x), b_{n}=\exp \left(z_{n}\right)$. We have

$$
\begin{aligned}
\exp [(\operatorname{Ad} g)(n x)+y] & =\exp [(\operatorname{Ad} g) n x] \exp y \\
& =g(\exp n x) g^{-1} h=g\left(\exp \left(y+z_{n}\right)\right) g^{-1} h \\
& =g h b_{n} g^{-1} h \in N h \cdot M \cdot N h \subseteq M .
\end{aligned}
$$

Thus $(\operatorname{Ad} g)(n x)+y \in W$ for large $n$; since $W$ is a wedge, $(\operatorname{Ad} g)(x)+(1 / n) y \in W$ for large $n$. Since $W$ is closed, $(\operatorname{Ad} g)(x) \in W$. Thus $\operatorname{Ad} g$ carries the interior of $W$ into $W$; because the interior is dense, $(\operatorname{Ad} g)(W) \subseteq W$. This is true for all $g \in N$, and since $N$ generates $G$ (by connectivity), it is true for all $g$ (since $\operatorname{Ad}\left(g_{1} \ldots g_{m}\right)=\operatorname{Ad} g_{1} \circ \cdots \circ \operatorname{Ad} g_{m}$ ).

\section{Frobenius-Perron groups}

In this section we let $V$ denote a finite dimensional vector space over $\mathbb{R}$. Let $G$ be a group, and let $\pi: G \rightarrow G L(V)$ be a continuous homomorphism. Then $\pi$ gives rise to a continuous linear action of $G$ defined by $g \cdot v=\pi(g)(v)$. E. B. Vinberg has shown that if $G$ is connected and solvable and preserves a pointed cone in $V$, then $G$ has a common eigenvector in the cone (see Lemma 1 of [27]). Indeed a stronger version occurs in the remarks following this lemma (for a more complete presentation, see Section I.7 of [12]).

Theorem 10.1 (Vinberg). Let $G$ be a Lie group, $S$ a solvable analytic subgroup, and $K$ a compact connected subgroup which normalizes $S$. If $C$ is a pointed cone invariant under the action of $G$, then there exists $v \in C$ with $K \cdot v=v$ and $S \cdot v \subseteq \mathbb{R}^{+} v$.

Theorem 10.1 motivates the following definition:

Definition 10.2. A topological group $G$ is called a Frobenius-Perron group if for every continuous linear action of $G$ on a finite dimensional vector space $V$ (over $\mathbb{R}$ ) that leaves a pointed cone $C$ invariant, there exists $v \in C$ with $G \cdot v \subseteq \mathbb{R}^{+} v$.

Let $V^{*}$ be the dual space of linear functionals from $V$ into $\mathbb{R}$, and define $\langle\rangle:, V^{*} \times V \rightarrow \mathbb{R}$ by $\langle\alpha, v\rangle=\alpha(v)$. Given a wedge $W \subseteq V$, we define $W^{*}=\left\{\alpha \in V^{*}:\langle\alpha, x\rangle \geqq 0\right.$ for all $x \in W\}$. This defines a duality of wedges (with $W^{* *}=W$ ). Also the left action of $G$ on $V$ gives rise to a unique right action on $V^{*}$ satisfying $(\alpha \cdot g, v\rangle=\langle\alpha, g \cdot v\rangle$. Via duality each theorem about actions (together with the duality of left and right actions by passing to inverses) gives rise to a dual theorem.

Now the dual of a pointed cone is a generating wedge (i.e., a wedge with interior) $W \neq V$, and the dual of a ray $\mathbb{R}^{+} v$ is a half-space. The dual of an invariant wedge is an invariant wedge (these assertions are all straightforward; see Chapter $I$ of [12] for details). Hence the dual version of Theorem 10.1 states 
Theorem 10.3. Let $G$ be a Frobenius-Perron group with a continuous linear action on a finite dimensional space $V$. If $W \neq V$ is an invariant generating wedge in $V$, then $W$ is contained in a half-space invariant under the action of $G$.

\section{Solvable groups}

In this section we use the previously developed theory (principally the nilpotent case, the Invariant Wedge Theorem, and Frobenius-Perron theory) to derive a major result of this paper: maximal subsemigroups with interior of connected solvable Lie groups are total.

A finite dimensional connected Lie group $G$ has a unique maximal connected solvable normal subgroup. It is denoted by $\operatorname{Rad} G$.

Theorem 11.1. Let $G$ be a finite dimensional connected Lie group which is a Frobenius-Perron group, and let $M$ be a maximal subsemigroup which is reduced in $G$ and satisfies int $M \neq \varnothing$. Then one of the following holds:

(i) $\operatorname{Rad} G=\{e\}$, i.e., $G$ is semisimple;

(ii) $(\operatorname{Rad} G, \operatorname{Rad} G \cap M)$ is topologically isomorphic to $\left(\mathbb{R}, \mathbb{R}^{+}\right)$;

(iii) $\operatorname{Rad} G$ is topologically isomorphic to $\mathrm{Aff}^{+}$.

Proof. If the radical $R$ of $G$ is trivial, then $G$ is semisimple.

We consider the case $R \neq\{e\}$, and show that either (ii) or (iii) results. The proof consists of a series of reductions.

1. The nil radical $N$ is abelian.

It is standard that the nil radical is the largest connected normal nilpotent subgroup. The assertion then follows immediately from Proposition 8.5.

2. The radical $R$ is metabelian (i.e. $[R, R]$ is abelian).

Again it is standard that $[R, R] \subseteq N$, which is abelian.

3. $\operatorname{dim}[R, R]=1$ or $[R, R]=\{e\}$.

Let $I$ be the Lie algebra for $[R, R]$. Since $[R, R]$ is normal and abelian, $I$ is an ideal in $L(G)$. Let $W=\{x \in I: \exp (x) \in M\}$. By the Invariant Wedge Theorem (9.1), $W$ is an invariant generating wedge. If $W=I$, then $\exp I=[R, R]$ (since $I$ is abelian) is a normal subgroup contained in $M$. Hence $[R, R]=\{e\}$.

If $W \neq I$, then since $G$ is a Frobenius-Perron group, there exists by Theorem 10.3 an invariant half-space $Q \supseteq W$. Then $Q \cap-Q$ is an invariant hyperplane in $I$, so $F=\exp (Q \cap-Q)$ is a normal subgroup. Now $\exp ^{-1}$ (int $\left.M\right) \cap I \subseteq$ int $W \subseteq$ int $Q \subseteq Q$ $\backslash(Q \cap-Q)$. Thus int $M \cap F=\varnothing$. By the Swallowing Lemma (3.4), $F \subseteq M$. Since $F$ is normal, $F \subseteq$ core $M=\{e\}$. Thus $Q \cap-Q=\{0\}$, so $I$ is one-dimensional, as is $\exp I=[R, R]$.

4. If $[R, R]=\{e\}$, then case (ii) obtains.

In this case $R$ is abelian. Let $J$ be the Lie algebra for $R$. We again obtain an invariant 
wedge $W \subseteq J$ since $M$ is reduced in $G$ and we are not in case (i). Thus as before one argues that $J$ is one-dimensional.

5. If $I=[L(R), L(R)]$ is one-dimensional, then the annihilator of $I$ in $L(R)$ is $I$.

Since $I$ is an ideal, its annihilator is easily verified to be an ideal. Hence $A$, the intersection of the annihilator with $L(R)$, is an ideal. Now $[A, A] \subseteq[L(R), L(R)] \subseteq I$; thus $[A,[A, A]] \subseteq[A, I]=\{0\}$. Thus $A$ is a nilpotent ideal, so $\exp A$ is a normal nilpotent group. It follows from Proposition 8.5 and the fact $M$ is reduced that $\exp A$ is abelian. Thus $A$ is abelian. An argument completely analogous to that applied to $I$ in step 3 yields $A$ is one-dimensional. Since $I \subseteq A, I=A$.

6. If $I=[L(R), L(R)]$ is one-dimensional, then $R$ is topologically isomorphic to $\mathrm{Aff}^{+}$.

Consider $\operatorname{Ad} z: L R) \rightarrow I=\mathbb{R} z$. By Reduction 5 the kernel of this mapping is $I$, which is one-dimensional. Thus $L(R)$ is two-dimensional. Since $[L(R), L(R)]=I \neq\{0\}, L(R)$ must be the unique non-abelian two-dimensional Lie algebra, so $R=\mathrm{Aff}^{+}$.

Corollary 11.2. Let $G$ be a finite dimensional connected solvable Lie group, and let $M$ be a maximal subsemigroup satisfying int $M \neq \varnothing$. Then $M$ is total and one of the following holds:

(i) $\left(G_{R}, M_{R}\right)$ is topologically isomorphic to $\left(\mathbb{R}, \mathbb{R}^{+}\right)$;

(ii) $\left(G_{R}, M_{R}\right)$ is topologically isomorphic to $\left(\mathrm{Aff}^{+}, \mathrm{Aff}^{++}\right)$.

Proof. By Theorem $10.1 G$ is a Frobenius-Perron group. Now $M_{R} \neq\{e\}$ (since $M_{R}$ is not a group), so case (ii) or (iii) of Theorem 11.1 must hold.

If $L\left(G_{R}\right)$ is the non-abelian two-dimensional Lie algebra, then $M_{R}$ does not contain the one-dimensional normal subgroup (since $M_{R}$ is reduced in $G_{R}$ ). By Proposition 6.5 $M$ is total and must be a "half-space" semigroup with boundary a non-normal group, and an inner automorphism then carries $M$ to the upper half-space $\mathrm{Aff}^{++}$.

\section{Extensions of solvable groups}

In this section we consider extensions of solvable groups by compact groups. Throughout this section $G$ denotes a finite dimensional connected real Lie group.

Lemma 12.1. The following are equivalent:

(1) $G / \operatorname{Rad} G$ is compact.

(2) The Levi subalgebras of $L(G)$ are compact (i.e., are the Lie algebras of some compact group).

(3) The analytic subgroups corresponding to the Levi subalgebras are compact.

(4) $G$ contains a connected solvable normal subgroup $H$ and a compact group $K$ such that $G=\bar{H} K$.

Proof. $(1) \Rightarrow(2)$ : The Levi subalgebras arise as cross-sections of the Lie algebra homomorphism from $L(G)$ to $L(G / \operatorname{Rad} G)$, and hence are isomorphic to $L G / \operatorname{Rad} G$ ). 
$(2) \Rightarrow(3)$ : If one of the Lie groups associated with a semisimple Lie algebra is compact, then they all are [17].

$(3) \Rightarrow(4): G=(\operatorname{Rad} G) \cdot K$ where $K$ is the analytic group associated with some Levi factor.

$(4) \Rightarrow(1)$ : Since $\operatorname{Rad} G$ is closed and is the largest connected solvable normal subgroup, $H \subseteq \operatorname{Rad} G$. Then the image of $K$ in $G / \operatorname{Rad} G$ must be all of $G / \operatorname{Rad} G$.

We extend Corollary 11.2 .

Theorem 12.2. Suppose $G / \operatorname{Rad} G$ is compact. If $M$ is a maximal subsemigroup of $G$ with int $M \neq \varnothing$, then $M$ is total, $\operatorname{Core}(M)$ is a normal, connected subgroup containing every semisimple analytic subgroup, and one of the following holds:

(i) $\left(G_{R}, M_{R}\right)$ is topologically isomorphic to $\left(\mathbb{R}, \mathbb{R}^{+}\right)$,

(ii) $\left(G_{R}, M_{R}\right)$ is topologically isomorphic to ( $\mathrm{Aff}^{+}, \mathrm{Aff}^{++}$).

Proof. If $\phi: G \rightarrow G_{R}$, then $\phi(\operatorname{Rad} G)=\operatorname{Rad} G_{R}$ (see Proposition 24 of 1II.9.7 [3]) so $G_{R} / \operatorname{Rad} G_{R}$ is homomorphic to $G / \operatorname{Rad} G$, hence compact. By Lemma 12.1 if $K$ is an analytic semisimple subgroup corresponding to some Levi factor of $L\left(G_{R}\right)$, then $K$ is compact and normalizes $\operatorname{Rad} G_{R}$. By Theorem $10.1, G_{R}$ is a Frobenius-Perron group. Hence we may apply Theorem 11.1 to $G_{R}$.

Case (i) is impossible, for then $G_{R}=K$ would be compact, an impossibility (see Proposition 5.7). Suppose case (ii) obtains, i.e., $\operatorname{Rad} G_{R}$ is topologically isomorphic to $\mathbb{R}$. Then $K$ acts on $\mathbb{R}$ by inner automorphisms, and hence must act trivially (since $K$ is compact and connected). Thus elements of $\mathbb{R}$ and $K$ commute, so $K$ is normal (since $G_{R}=\mathbb{R} K$ ). Then Proposition 5.7 implies $K=\{e\}$ (since $M_{R}$ is reduced). So $G_{R}=\operatorname{Rad} G_{R}$. Since the image of any analytic semisimple subgroup of $G$ must be a semisimple subgroup of $\mathbb{R}$, the image must be trivial. Theorem 11.1 implies the rest.

Suppose finally that the case $\operatorname{Rad} G_{R}$ is topologically isomorphic to Aff ${ }^{+}$of Theorem 11.2 obtains. Again $K$ must act on Aff ${ }^{+}$by inner automorphisms. But Aut (Aff ${ }^{+}$) contains no non-trivial compact connected subgroups. (Its identity component is again topologically isomorphic to Aff ${ }^{+}$, since every derivation is inner (see [18]), and Aff ${ }^{+}$ contains no non-trivial compact subgroup.) Thus again $K$ acts trivially, is thus normal, and hence $K=\{e\}$. So $G_{R}=\operatorname{Rad} G_{R}$. Since $M_{R}$ is closed and maximal in $G_{R}$, it must be topologically isomorphic to Aff ${ }^{++}$by Corollary 11.2.

Finally Lemma 7.1 implies that $\operatorname{Core}(M)$, the kernel of $\phi$, is connected.

Remark 12.3. Theorem 12.2 actually extends to locally compact connected groups which contain a compact normal subgroup $K$ such that $G / K$ is a Lie group with $G / \operatorname{Rad} G$ compact. (For by Proposition 5.7 it must be the case that $K \subseteq \operatorname{Core}(M)$ for any maximal subsemigroup $M$ with $\operatorname{int}(M) \neq \varnothing$. Then apply the theorem to $G / K$ and $M / K$.)

Corollary 12.4. Suppose $G / \operatorname{Rad} G$ is compact. If $G$ contains a subsemigroup $S \neq G$ with int $S \neq \varnothing$, then one of the following holds:

(i) There exists a continuous homomorphism $\phi: G \rightarrow \mathbb{R}$ such that $\phi(S) \subseteq \mathbb{R}^{+}$. 
(ii) There exists a continuous homomorphism $\phi: G \rightarrow \mathrm{Aff}^{+}$such that $\phi(S) \subseteq \mathrm{Aff}^{++}$.

Proof. Extend $S$ to a maximal subsemigroup $M$ and apply Theorem 12.2.

The next corollary gives a classification of maximal semigroups in terms of the Lie algebra for the simply connected case.

Theorem 12.5. The maximal subsemigroups $M$ with non-empty interior of a simply connected Lie group $G$ with $G / \operatorname{Rad} G$ compact are in one-to-one correspondence with their tangent objects

$$
L(M)=\{x \in L(G): \exp t x \in M \text { for } t \geqq 0\}
$$

and the latter are precisely the closed half-spaces with boundary a subalgebra. Furthermore, $M$ is the semigroup generated by $\exp (L(M))$.

Proof. Let $M$ be maximal with $\operatorname{int}(M) \neq \varnothing$. By Theorem $12.2 G_{R}$ is either $\mathbb{R}^{\text {or } \text { Aff }^{+}}$ and $\operatorname{Core}(M)$ is connected. Hence $\operatorname{Core}(M)$ is generated by the exponential image of its tangent subalgebra. One verifies directly in each case that $M_{R}$ is (hence is generated by) the exponential of its tangent set. It follows from commuting diagrams of Lie algebras and groups that $M$ is generated by exp of its tangent set. Also since $M_{R}$ has tangent set a wedge with a bounding subalgebra of codimension 1, this property pulls back and remains valid for $M$.

Conversely suppose $G$ is simply connected and $A$ is a half-space bounded by a subalgebra $Q$ of codimension 1 . Then $Q$ is an ideal or contains an ideal of codimension 2 or 3 (see [13] or [26]), and there exists a Lie algebra homomorphism $L(\phi)$ either onto $\mathbb{R}$ with kernel $Q$ or onto the non-abelian two-dimensional Lie algebra or onto $\operatorname{sl}(2, \mathbb{R})$. But the last case is impossible since than $L(\operatorname{Rad} G)$ must necessarily map to $\{0\}$, and then $\operatorname{sl}(2, \mathbb{R})$ would be the image of a compact Lie algebra (Lemma 12.1), hence compact, a contradiction. Also $L(\phi)(A)$ will be a half-space in $\mathbb{R}$ or $L\left(\mathrm{Aff}^{+}\right)$. Since $G$ is simply connected, there is a corresponding $\phi: G \rightarrow \mathbb{R}$ or $\phi: G \rightarrow \mathrm{Aff}^{+}$. Pulling back the subsemigroups of $\mathbb{R}$ or $\mathrm{Aff}^{+}$corresponding to $L(\phi)(A)$, one obtains a maximal subsemigroup $M$ of $G$ containing $\exp (A)$. Since $A \subseteq L(M) \neq L(G)$ and $A$ is a half-space, $A=L(M)$.

Corollary 12.6. Let $\Omega$ generate (as a Lie algebra) $L(G)$, the Lie algebra of $G$, where $G / \operatorname{Rad} G$ is compact. If $\Omega$ is not contained in any half-space with boundary a subalgebra, then the semigroup generated by $\exp \left(\mathbb{R}^{+} \Omega\right)$ is all of $G$. The converse holds if $G$ is simply connected.

Proof. Let $S$ be the semigroup generated by $\exp \left(\mathbb{R}^{+} \Omega\right)$. Since $\Omega$ generates $L(G)$, it is standard that int $S \neq \varnothing$ (see [25]). If $S \neq G$, extend $S$ to a maximal subsemigroup $M$. Then $\mathbb{R}^{+} \Omega \subseteq L(S) \subseteq L(M)$, and by Theorem 12.5 the latter is a half-space with bounding subalgebra (simple connectivity was not used for this part of the proof). But this is a contradiction, so $S=G$. The converse follows similarly using Theorem 12.5 . 
We remark that the finite dimensionality assumption is necessary in order to have a Frobenius-Perron theory for solvable groups. One obtains in general such a theory for compact groups, and hence a version of Theorem 12.2 (and the other theorems) could be carried out for infinite-dimensional Lie groups for the case $G$ was the product of a compact group and a normal connected nilpotent Lie group.

\section{Maximal semigroups of mixed type}

Let $G$ be a finite dimensional connected Lie group. The maximal subsemigroups of $G$ may be divided into three classes. We have those in which $\operatorname{Core}(M)$ contains one (and hence all) of the maximal semisimple analytic subgroups. This is the case that arises in the previous section. We have seen that $M$ is total, is generated by the exponential image of a half-space with a bounding subalgebra of codimension 1 , and is the inverse image of a maximal subsemigroup of a solvable group. We call $M$ a maximal semigroup of solvable type.

A second possibility is that $G$ admits a continuous homomorphism onto a semisimple Lie group $E$ and that $M$ is the inverse image of a maximal subsemigroup in $E$ (and hence maximal by the Reduction Lemma). Equivalenily $M$ (and hence $\operatorname{Core}(M)$ ) contains $\operatorname{Rad} G$. In this case $M$ is called a maximal subsemigroup of semisimple type. To study these, one needs a classification of maximal subsemigroups which are reduced in semisimple Lie groups. Practically nothing is known in this case. Hilgert and Hofmann [9] have characterized the maximal subsemigroups for groups associated with $\operatorname{sl}(2, \mathbb{R})$, and sufficient conditions are given in [19] for a subset of the Lie algebra to generate (as a semigroup) the entire group. This is certaintly an area that should be given top priority in the future study of maximal subsemigroups.

A third type of maximal subsemigroups are those of mixed type, where $\operatorname{Core}(M)$ contains neither $\operatorname{Rad}(G)$ nor a maximal semisimple analytic subgroup. We give a construction that gives rise to examples of mixed type.

Let $V$ be a finite dimensional real vector space and let $G$ be a connected Lie group that acts linearly on $V$. We form the semidirect product $V \times G$ with multiplication defined by $\left(v_{1}, g_{1}\right)\left(v_{2}, g_{2}\right)=\left(v_{1}+g_{1} \cdot v_{2}, g_{1} g_{2}\right)$.

Proposition 13.1. Let $W$ be a wedge in $V$ with $W \neq V, W-W=V$. If $W$ is a maximal invariant wedge in $V$ under the action of $G$, then $S=W \times G$ is a maximal subsemigroup of $V \times G$.

Proof. It is immediate that $S$ is a closed subsemigroup with nun-empty interior. Hence it is contained in a maximal subsemigroup $M$ (Proposition 5.2). We may identify $L(V \times G)$ with $V \times L(G)$; then by the Invariant Wedge Theorem (9.1), $M \cap V$ is an invariant wedge. Clearly $M \cap V \neq V$ (otherwise $M \supseteq V \cdot S=V \times G$ ). Thus $M \cap V=W$ since $W \subseteq M \cap V$ and $W$ is a maximal invariant wedge. If $(v, g) \in M$, then $(v, g)\left(0, g^{-1}\right)=$ $(v, e) \in M$. Thus $v \in W$ and hence $(v, g) \in W \times G=S$. We conclude $M=S$, i.e., $S$ is maximal.

Invariant cones for irreducible actions of semisimple Lie groups $G$ have been studied by Vinberg [27]. In particular it is shown that invariant cones exist in $V$ if and only if 
there is a non-zero vector fixed by $K$, a maximal compact subgroup of $G$. In this case every invariant cone is contained in a maximal invariant cone. Thus this construction gives rise to many examples.

It is conjectural that the preceding construction should give rise to all maximal semigroups with non-empty interior of mixed type for the case $G$ is a connected Lie group with $\operatorname{Rad} G$ nilpotent, and that some variant thereof should hold in general. The theory of invariant cones as developed by Vinberg [27], Ol'shanskii [20, 21], and Paneitz $[23,24]$ should play a crucial role in the theory of both maximal semigroups of semisimple and mixed type.

\section{REFERENCES}

1. B. Bonnard, V. Jurdjevic, I. Kupka and G. Sallet, Transitivity of families of invariant vector fields on the semidirect product of Lie groups, Trans. Amer. Math. Soc. 271 (1982), 525-535.

2. N. Bourbaki, Topologie generale (Hermann, Paris, 1958).

3. N. Bourbaki, Lie Groups and Lie Algebras, I (Addison-Wesley, Reading, 1975).

4. A. H. Clifford and G. B. Preston, The Algebraic Theory of Semigroups, I (Amer. Math. Soc., 1961).

5. J. G. Dobbins, Well-bounded semigroups in locally compact groups, Math. Z. 148 (1976), 155-167.

6. L. Fuchs, Partially Ordered Algebraic Systems (Pergamon Press, 1963).

7. J. Gauthier, I. KuPKA and G. SAllet, Controllability of right invariant systems on real simple Lie groups (Preprint Institut Fourier, 1984).

8. J. HiLgert, Maximal semigroups and controllability in products of Lie groups (Preprint THD Nr. 971, 1986).

9. J. Hilgert and K. HofmanN, Old and new on SL(2), Manuscripta Math. 54 (1985), 17-52.

10. J. Hilgert and K. Hofmann, On Sophus Lie's Fundamental Theorems, J. Funct. Anal., to appear.

11. J. Hilgert, K. Hofmann and J. D. Lawson, Controllability of systems on a nilpotent Lie group, Beiträge Algebra Geom. 30 (1985), 185-190.

12. J. Hilgert, K. Hofmann and J. D. Lawson, The Lie Theory of Semigroups (Monograph, in preparation).

13. K. Hofmann, Lie algebras with subalgebras of codimension one, Illinois J. Math. 9 (1965), $636-643$.

14. K. Hofmann and J. Lawson, Foundations of Lie Semigroups (Lecture Notes in Math. 998, 1983), 128-201.

15. K. Hofmann and J. Lawson, On Sophus Lie's Fundamental Theorems, I, Indag. Math. 45 (1983), 453-466.

16. K. Hofmann and J. D. Lawson, On Sophus Lie's Fundamental Theorems II, Indag. Math. 46 (1984), 255-265.

17. G. Hocrschild, The Structure of Lie Groups (Holden Day, San Francisco, 1965).

18. N. JACOBSON, Lie Algebras (Dover, New York, 1962).

19. V. Jurdevic and I. KuPKa, Control systems on semisimple Lie groups and their homogeneous spaces, Ann. Inst. Fourier 31 (1981), 151-179. 
MAXIMAL SUBSEMIGROUPS OF LIE GROUPS THAT ARE TOTAL 501

20. G. Olshanskil, Convex cones in symmetric Lie algebras, Lie semigroups, and invariant causal (order) structures on pseudo-Riemannian symmetric spaces, Soviet Math. Dokl. 26 (1982), 97-101.

21. G. Ol'shanskil, Invariant cones in symmetric Lie algebras, Lie semigroups, and the holomorphic discrete series, Functional Anal. Appl. 15 (1981), 275-285.

22. S. PANeITZ, Invariant convex cones and causality in semisimple Lie algebras and groups. $J$. Funct. Anal. 43 (1981), 313-359.

23. S. Paneitz, Classification of invariant convex cones in simple Lie algebras, Ark. Mat. 21 (1984), 217-228.

24. D. Poguntke, Well-bounded semigroups in connected groups, Semigroup Forum 15 (1977), 159-167.

25. H. Sussman and V. Jurdevic, Controllability of nonlinear systems, J. Differential Equations 12 (1972), 95-116.

26. J. Tirs, Sur une classe de groupes de Lie resolables, Bull Soc. Math. Belg. 11 (1959), $100-115$.

27. E. Vingerg, Invariant cones and orderings in Lie groups, Functional Anal. Appl. 14 (1980), $1-13$.

28. F. WRIGHT, Semigroups in compact groups, Proc. Amer. Math. Soc. 7 (1956), 309-311.

29. F. WRIGHT, Topological abelian groups, Amer. J. Math. 79 (1957), 477-496.

30. F. WRIGHT, Holder groups, Duke Math. J. 24 (1957), 567-571.

Department of Mathematics

Louisiana State University

Baton Rouge

LOUISIANA 70803

U.S.A. 